\title{
Association between neuropeptide $Y$ receptor Y2 promoter variant rs6857715 and major depressive disorder Jens Treutlein ${ }^{a, \star}$, Jana Strohmaier ${ }^{a, \star}$, Josef Frank ${ }^{a}$, Stephanie H. Witt ${ }^{a}$, Liz Rietschel $^{\mathrm{d}}$, Andreas J. Forstner ${ }^{\mathrm{b}, \mathrm{c}}$, Maren Lang ${ }^{\mathrm{a}}$, Franziska Degenhardt ${ }^{\mathrm{b}, \mathrm{c}}$, Helene Dukal ${ }^{\mathrm{a}}$, Stefan Herms ${ }^{\mathrm{b}, \mathrm{c}, \mathrm{e}}$, Fabian Streit ${ }^{\mathrm{a}}$, Per Hoffmann ${ }^{\mathrm{b}, \mathrm{c}, \mathrm{e}}$, Sven Cichon ${ }^{\mathrm{b}, \mathrm{c}, \mathrm{e}}$, Markus M. Nöthen ${ }^{\mathrm{b}, \mathrm{c}}$ and Marcella Rietschel ${ }^{\mathrm{a}}$
}

\begin{abstract}
Stress increases the risk for major depressive disorder (MDD), overeating, and alcohol dependence (AD). The neuropeptide $Y$ system is one of the best-known modulators of the stress response, and some of its effects are mediated through the neuropeptide $Y$ receptor $Y 2$ (NPY2R). The functional NPY2R variant rs6857715 (C-599T) has been implicated in both obesity and AD, but with opposing alleles. The present study explored whether rs6857715 is also associated with MDD. Analysis of the overall sample (595 MDD cases; 1295 controls) showed an association with the $A D$ risk allele $C[P=0.020$, odds ratio $(O R)(C$-allele $)=1.18]$. The association remained significant after excluding MDD patients with AD/alcohol abuse $[P=0.038$, OR $(C$-allele $)=1.18]$; increased weight/appetite $[P=0.006$, OR $(C$-allele $)=1.23]$; or both $[P=0.008$, OR (Callele) $=1.25$ ]. The present findings suggest that the NPY2R rs6857715 C-allele makes a genuine contribution toward MDD. Psychiatr Genet 27:34-37 Copyright (C) 2017 Wolters Kluwer Health, Inc. All rights reserved.
\end{abstract}

Psychiatric Genetics 2017, 27:34-37

Keywords: appetite increase, C-599T, major depressive disorder, neuropeptide $\mathrm{Y}$ receptor $\mathrm{Y} 2, N P Y 2 R$, rs6857715, weight gain

aDepartment of Genetic Epidemiology in Psychiatry, Central Institute of Mental Health, Medical Faculty Mannheim, Heidelberg University, Heidelberg, 'Institute of Human Genetics, University of Bonn, Bonn, Germany, 'Department of Genomics, Life and Brain Center, University of Bonn, Bonn, Germany, ${ }^{d}$ Department of Child and Adolescent Psychiatry, University Psychiatric Hospital, Bern, Switzerland and ${ }^{\text {e}}$ Department of Biomedicine, Division of Medical Genetics, University of Basel, Basel, Switzerland

Correspondence to Marcella Rietschel, MD, Department of Genetic Epidemiology in Psychiatry, Central Institute of Mental Health, Faculty of Medicine Mannheim, University of Heidelberg, J5, 65189 Mannheim, Germany Tel: + 4962117036051 ; fax: + 4962117036055 ; e-mail: marcella.rietschel@zi-mannheim.de

*Jens Treutlein and Jana Strohmaier contributed equally to the writing of this article.

Received 2 March 2016 Revised 29 July 2016 Accepted 4 August 2016

\section{Introduction}

Stress increases the risk for major depressive disorder (MDD), alcohol dependence (AD), and obesity (Kendler et al., 1999; Sinha and Jastreboff, 2013). These conditions manifest in both isolated and comorbid states. Here, epidemiological studies have reported comorbidity between MDD and AD (Petrakis et al., 2002; Hasin et al., 2005) and between MDD and obesity (Petry et al., 2008; de Wit et al., 2010). In humans and animals, one of the most widely reported mediators of resilience to stress is neuropeptide $\mathrm{Y}$ (NPY) (Hirsch and Zukowska, 2012; Sah and Geracioti, 2013). NPY exerts some of its effects through the neuropeptide $\mathrm{Y}$ receptor $\mathrm{Y} 2(N P Y 2 R)$. Genetic variation in $N P Y 2 R$ may thus determine individual response to stress and the propensity to stress-induced disorders (Enman

\footnotetext{
Supplemental digital content is available for this article. Direct URL citations appear in the printed text and are provided in the HTML and PDF versions of this article on the journal's website (www.psychgenetics.com).

This is an open-access article distributed under the terms of the Creative Commons Attribution-Non Commercial-No Derivatives License 4.0 (CCBY-NCND), where it is permissible to download and share the work provided it is properly cited. The work cannot be changed in any way or used commercially without permission from the journal.
}

et al., 2015). Genetic studies have reported an association between a functional variant in the $N P Y 2 R$ promoter (rs6857715; synonym: C-599T; higher expression reported for the rs6857715 T-allele (Wei et al., 2013)) and both obesity (Siddiq et al., 2007) and AD [Frank et al., 2012 (rs6857715 $P=0.005$, odds ratio $(\mathrm{OR})(\mathrm{C}$-allele $)=1.16)$; Wetherill et al., 2008]. For further information, see Supplementary Text, Supplemental digital content 1 (http://links.lww.com/PG/A167) and Supplementary figs S2, Supplemental digital content 1 (http://links.lww.com/PG/ A167); S3, Supplemental digital content 1 (http://links.lww. com/PG/A167); S4, Supplemental digital content 1 (http:// links.lww.com/PG/A167); and S5, Supplemental digital content 1 (http://links.lww.com/PG/A167). However, risk was conferred by the $\mathrm{T}$-allele in obesity and the C-allele in AD. To our knowledge, no candidate study to date has tested whether rs6857715 is also associated with MDD.

The aim of the present study were two-fold. First, we investigated whether the NPY2R variant rs6857715 is associated with MDD. Second, we investigated whether this association is genuine or instead because of subgroups of MDD patients with comorbid AD/alcohol 
abuse or increased appetite/weight. Given the previously reported association of the C-allele with $\mathrm{AD}$ and of the T-allele with obesity, we hypothesized that MDD patients with $\mathrm{AD} /$ alcohol abuse would show increased C-allele frequency, whereas MDD patients with increased appetite/weight would show increased T-allele frequency in comparison with MDD patients without these comorbid conditions.

\section{Materials and methods Participants}

The present German sample comprised 595 MDD patients and 1295 population-based controls. Data on AD/alcohol abuse were available for 537 MDD patients and 56 MDD patients showed one of these disorders. Data on appetite increase and/or weight gain were available for 594 MDD patients and 84 MDD patients showed one of these features. Thirteen MDD patients showed both $\mathrm{AD} /$ alcohol abuse and increased weight/ appetite.

In patients, clinical symptoms and lifetime 'best estimate' diagnoses of MDD, AD, and alcohol abuse were assessed using multiple sources of information including the Structured Clinical Interview for Diagnostic and Statistical Manual of Mental Disorders, 4th ed. (DSM-IV) Axis I Disorders (First et al., 1994); medical records; and information obtained using the family history method. The items 'appetite increase' and 'weight gain' were assessed using the Structured Clinical Interview for DSM-IV Axis I Disorders and patients were asked to indicate whether or not they considered these changes to have been secondary to medication. The items were only rated as present in individuals for whom appetite increase and/or weight gain could be attributed to the diagnosis per se and not to medication. Diagnosis was assigned according to the DSM-IV criteria. In each case, a consensus diagnosis was assigned by two raters. All patients had a history of at least one episode of MDD severe enough to warrant hospitalization.

The study was approved by the respective local ethics committees and all participants provided written informed consent before inclusion.

\section{Single nucleotide polymorphism selection and genotyping}

The NPY2R promoter variant rs6857715 was selected as it is reported to confer allele-specific expression differences in vitro (Wei et al., 2013). In addition, rs6857715 has been associated with $\mathrm{AD}$ and obesity (Siddiq et al., 2007; Wetherill et al., 2008; Frank et al., 2012; Supplementary Text, Supplemental digital content 1, http://links.lww.com/ $P G / A 167$ and Supplementary fig. S5, Supplemental digital content 1, http://links.kww.com/PG/A167). Detailed information on genotyping is provided in Rietschel et al. (2010). Experimental genotyping cluster plots for rs6857715 are shown in Supplementary fig. S1, Supplemental digital content 1 (http://links.lww.com/PG/A167).

\section{Statistical analysis}

Power calculations were performed using the Genetic Power Calculator (Purcell et al., 2003; http://pngu.mgh.har vard.edu/ purcell/gpc/cc2.html) using a multiplicative model of inheritance; a disease/marker allele frequency of 0.61 (1000 Genomes CEU reference sample; http://www.ncbi. nlm.nih.gov/projects/SNP/snp_ref.cgi? $r s=6857715)$; a lifetime prevalence of major depressive episodes in Germany of around 10\% (Kessler and Bromet, 2013); a control: case ratio of $2.18\left(D^{\prime}=1\right)$ between disease and marker allele; and a significance level of $\alpha=0.05$. The power of the sample to detect association with MDD ranged from 53 to more than $80 \%$ depending on the proposed effect size. For a proposed effect size of $\mathrm{OR}$ (C-allele $)=1.16$, as based on the association with AD (Frank et al., 2012), the power was $53 \%$. For a proposed effect size of OR (T-allele) $=1.345$, as based on the association with obesity (Siddiq et al., 2007), the power was 98\%. In Siddiq et al. (2007), the OR was calculated from the genotype counts of 204/208/67 obese adults and 475/378/85 controls. The minimum effect size detectable in the present sample is $\mathrm{OR}=1.23$.

Deviation from Hardy-Weinberg equilibrium (HWE) was calculated using an exact test (http://ihg.gsf.de/cgi-bin/ hw/hwa1.pl). Association testing for rs6857715 was performed using the Armitage Trend Test (http://ihg.gsf.del cgi-bin/hw/hwa1.pl).

\section{Results}

Genotype counts, allele frequencies, and deviation from HWE results are shown in Table 1 (Supplementary fig. S4, Supplemental digital content 1, http://links.lww.com/ $P G / A 167)$. Distribution of genotypes did not deviate from HWE (Table 1).

Comparison of all MDD patients $(N=595)$ versus controls $(N=1295)$ showed a significant association between MDD and the C-allele of rs6857715 $[P=0.020$, OR (Callele $)=1.18$. This association remained significant when the analysis was restricted to MDD patients without $\mathrm{AD} /$ alcohol abuse versus controls $[P=0.038$, OR (Callele $=1.18]$; MDD patients without increased weight $/$ appetite versus controls $[P=0.006, \mathrm{OR}(\mathrm{C}$-allele $)=1.23]$; and MDD patients with neither $\mathrm{AD} / \mathrm{alcohol}$ abuse nor increased weight/appetite versus controls $[P=0.008$, OR $(\mathrm{C}$-allele $)=1.25]$. No significant association was found in a case-only analysis of MDD patients without $\mathrm{AD} / \mathrm{alcohol}$ abuse versus MDD patients with $\mathrm{AD} /$ alcohol abuse $[P$ (one sided $)=0.302$, OR $(\mathrm{C}$-allele $)=1.11 \mathrm{j}$. Comparison of MDD patients without increased weight/appetite versus MDD patients with increased weight/appetite almost reached significance $[P$ (one sided $)=0.054$, OR $(\mathrm{T}$ allele $)=1.31]($ Table 2$)$. 
Table 1 Number of individuals per group, rs6857715 genotype counts, allele frequencies, and deviation from Hardy-Weinberg equilibrium

\begin{tabular}{|c|c|c|c|c|}
\hline Group/subgroup & Number of individuals & Genotype counts (CC, CT, TT) & Allele frequency $(\mathrm{C})$ & $\mathrm{HWE}^{\mathrm{c}}$ \\
\hline MDD patients & 595 & $240,279,76$ & 0.64 & 0.79 \\
\hline MDD patients with appetite increase/weight gain ${ }^{a}$ & 84 & $30,38,16$ & 0.58 & 0.51 \\
\hline MDD patients without appetite increase/weight gain ${ }^{a}$ & 510 & $210,240,60$ & 0.65 & 0.56 \\
\hline MDD patients with $A D / a l c o h o l ~ a b u s e^{b}$ & 56 & $25,24,7$ & 0.66 & 0.77 \\
\hline MDD patients without $A D /$ alcohol abuse ${ }^{b}$ & 481 & $191,230,60$ & 0.64 & 0.49 \\
\hline $\begin{array}{l}\text { MDD patients without appetite increase/weight gain and without } A D / a \text { alcohol } \\
\text { abuse (MDD only) }\end{array}$ & 414 & $171,196,47$ & 0.65 & 0.45 \\
\hline Population-based controls & 1295 & $465,619,211$ & 0.60 & 0.86 \\
\hline
\end{tabular}

AD, alcohol dependence; HWE, Hardy-Weinberg equilibrium; MDD, major depressive disorder.

${ }^{2}$ Data were available for 594 of the 595 patients.

bData were available for 537 of the 595 patients.

${ }^{\mathrm{c}} \mathrm{HWE}$ exact test $P$-values (according to http://ihg.gsf.de/cgi-bin/hw/hwa1.p/).

Table $2 P$-values and effect sizes

\begin{tabular}{|c|c|c|}
\hline Comparison groups/subgroups & $P$-value ${ }^{\mathrm{a}}$ & Effect size ${ }^{b}$ \\
\hline MDD patients versus population-based controls & $P=0.020$ (two sided) & $\begin{array}{l}\text { OR }(\text { C-allele })=1.18 \\
\mathrm{Cl}=1.03-1.36\end{array}$ \\
\hline MDD patients without appetite increase/weight gain versus population-based controls & $P=0.006$ (two sided) & $\begin{array}{l}\text { OR }(\mathrm{C} \text {-allele })=1.23 \\
\mathrm{Cl}=1.06-1.43\end{array}$ \\
\hline MDD patients without $\mathrm{AD} / \mathrm{alcohol}$ abuse versus population-based controls & $P=0.038$ (two sided) & $\begin{array}{l}\text { OR }(\mathrm{C} \text {-allele })=1.18 \\
\mathrm{Cl}=1.01-1.37\end{array}$ \\
\hline $\begin{array}{l}\text { MDD patients without appetite increase/weight gain and without AD/alcohol abuse versus population-based controls } \\
\text { (MDD only) }\end{array}$ & $P=0.008$ (two sided) & $\begin{array}{l}\text { OR }(\mathrm{C} \text {-allele })=1.25 \\
\mathrm{Cl}=1.06-1.47\end{array}$ \\
\hline MDD patients with appetite increase/weight gain versus MDD patients without appetite increase/weight gain & $\begin{array}{l}P=0.109 \text { (two sided) } \\
P=0.054 \text { (one sided) }\end{array}$ & $\begin{array}{l}\text { OR }(\text { T-allele })=1.31 \\
\mathrm{Cl}=0.94-1.83\end{array}$ \\
\hline MDD patients with $A D /$ alcohol abuse versus MDD patients without $A D /$ alcohol abuse & $\begin{array}{l}P=0.604 \text { (two sided) } \\
P=0.302 \text { (one sided) }\end{array}$ & $\begin{array}{c}\text { OR }(\text { C-allele })=1.11 \\
\mathrm{Cl}=0.74-1.68\end{array}$ \\
\hline
\end{tabular}

$\mathrm{AD}$, alcohol dependence; $\mathrm{Cl}$, confidence interval; MDD, major depressive disorder; OR, odds ratio.



${ }^{\mathrm{b}}$ Allelic OR and $\mathrm{Cl}$ (according to http://ihg.gsf.de/cgi-bin/hw/hwa1.pl).

\section{Discussion}

The present analyses showed a significant association between MDD and the C-allele of the NPY2R promoter variant rs6857715, that is, the previously reported risk allele for AD. Exclusion of subgroups showed that this association is genuinely attributable to MDD and not to comorbid AD/alcohol abuse or increased weight/appetite. As hypothesized, the MDD patient group with increased weight/appetite showed a higher $\mathrm{T}$-allele frequency than the MDD patient group without increased weight/ appetite. This difference just failed to reach significance $[P($ one sided $)=0.054]$, which may have been attributable to the small sample size. However, if a large proportion of our MDD patients had had increased weight/ appetite, the contrasting allelic effects on MDD and weight/appetite might have obscured the detection of any association.

A significant change in weight and/or appetite is a key diagnostic feature of MDD. Although significant loss of weight and/or decrease in appetite are typical features of so-called endogenous depression (Paykel, 2008), increased weight/appetite are reported by more than $40 \%$ of patients with atypical depression (Sullivan et al., 1998; Thase, 2007). Research has shown that atypical depression is the most common form of depression in outpatient psychiatry (Nierenberg et al., 1998; Singh and Williams, 2006). The term 'atypical' is used to distinguish this form of illness from the more severe 'endogenous' depression phenotype, which was the norm at the time when depression was rarely diagnosed in outpatients (American Psychiatric Association, 2013). The concept of endogenous versus reactive/atypical depression has been the subject of contentious debate for decades and has been abandoned because of lack of consistent biological support and therapeutic implications (Paykel, 2008). However, clearly measurable differences between patient subgroups, such as weight gain/loss, could facilitate dissection of clinical heterogeneity for the purposes of genetic studies.

As differences in the peptide NPY regulate both stress and food intake (Maniam and Morris, 2012), T-allele carriers may constitute a specific group of patients who are more likely to react to stress (e.g. stressful life events and/or MDD) by increased appetite/weight gain than is the case for C-allele carriers. Animal studies support this hypothesis as they have shown that stress upregulates $N p y 2 r$ in a glucocorticoid-dependent manner and that upregulated Npy2r stimulates fat angiogenesis, leading to a $50 \%$ increase in visceral fat within a 2-week period. In contrast, Npy2r pharmacological inhibition or knockdown prevents the accumulation of fat under stress (Kuo et al., 2007, 2008). The higher NPY2R expression reported for the T-allele (Wei et al., 2013) is consistent with such a hypothesis. To investigate the role of the T-allele in atypical depression, large longitudinal studies of stress are warranted. 


\section{Conclusion}

The present findings in MDD patients with a history of inpatient treatment support the hypothesis that the NPY2R variant rs6857715 is implicated not only in $\mathrm{AD}$ and obesity but also in MDD. Furthermore, our findings suggest that homogeneity in terms of MDD symptoms such as weight gain may facilitate genetic research into depression. In this context, it would be of interest to include information on appetite/weight increase in the Psychiatric Genomics Consortium MDD genome-wide association study analysis. In a previous mega-analysis, the Psychiatric Genomics Consortium included both inpatients and outpatients, and identified no association between this variant and the categorical diagnosis MDD $(P=0.590$; Major Depressive Disorder Working Group of the Psychiatric GWAS Consortium et al., 2013; http://wwew.med.unc.edu/pgc/downloads). Further investigation of stress-related phenotypes may generate insights into the mechanisms acting across somatic and psychiatric diagnoses.

\section{Acknowledgements}

The study was supported by the German Federal Ministry of Education and Research (BMBF) within the context of the following projects: (i) the Integrated Genome Research Network (IG) MooDS of the German National Genome Research Network plus (NGFNplus) (grant 01GS08144 to Sven Cichon and Markus M. Nöthen; grant 01GS08147 to Marcella Rietschel); (ii) the Integrated Network IntegraMent (Integrated Understanding of Causes and Mechanisms in Mental Disorders; grant 01ZX1314A to Sven Cichon and Markus M. Nöthen, grant 01ZX1314G to Marcella Rietschel); and (iii) SysMedAlcoholism (Alcohol Addiction: A Systems-Oriented Approach, grant 01ZX1311A). The latter two projects are being conducted under the auspices of the BMBF e:Med Systems Medicine Programme. Support was also provided by the German Research Foundation (DFG Exzellenzinitiative II to J.S. and DFG TR 920/2-1 to J.T.). Markus M. Nöthen is a member of the DFG-funded Excellence-Cluster ImmunoSensation. Marcella Rietschel and Stephanie H. Witt are members of the Collaborative Research Center SFB636 of the University of Heidelberg. The study was also supported by the German Research Foundation (DFG; grant FOR2107; RI908/11-1 to M.R.; NO246/10-1 to M.M.N.).

\section{Conflicts of interest}

There are no conflicts of interest.

\section{References}

American Psychiatric Association (2013). Diagnostic and Statistical Manual of Mental Disorders, 5th ed. Arlington, VA: American Psychiatric Association.

De Wit L, Luppino F, van Straten A, Penninx B, Zitman F, Cuijpers P (2010). Depression and obesity: a meta-analysis of community-based studies. Psychiatry Res 178:230-235.
Enman NM, Sabban EL, McGonigle P, van Bockstaele EJ (2015). Targeting the neuropeptide $Y$ system in stress-related psychiatric disorders. Neurobiol Stress 1:33-43.

First MB, Spitzer RL, Gibbon M, Williams JBW (1994). Structured Clinical Interview for DSM-IV Axis I Disorders. New York: New York State Psychiatric Institute, Biometrics Research.

Frank J, Cichon S, Treutlein J, Ridinger M, Mattheisen M, Hoffmann P (2012). Genome-wide significant association between alcohol dependence and a variant in the ADH gene cluster. Addict Biol 17:171-180.

Hasin DS, Goodwin RD, Stinson FS, Grant BF (2005). Epidemiology of major depressive disorder: results from the National Epidemiologic Survey on Alcoholism and Related Conditions. Arch Gen Psychiatry 62:1097-1106.

Hirsch D, Zukowska Z (2012). NPY and stress 30 years later: the peripheral view. Cell Mol Neurobiol 32:645-659.

Kendler KS, Karkowski LM, Prescott CA (1999). Causal relationship between stressful life events and the onset of major depression. Am J Psychiatry 156:837-841.

Kessler RC, Bromet EJ (2013). The epidemiology of depression across cultures. Annu Rev Public Health 34:119-138.

Kuo LE, Kitlinska JB, Tilan JU, Li L, Baker SB, Johnson MD, et al. (2007). Neuropeptide $Y$ acts directly in the periphery on fat tissue and mediates stress-induced obesity and metabolic syndrome. Nat Med 13:803-811.

Kuo LE, Czarnecka M, Kitlinska JB, Tilan JU, Kvetnanský R, Zukowska Z (2008). Chronic stress, combined with a high-fat/high-sugar diet, shifts sympathetic signaling toward neuropeptide $\mathrm{Y}$ and leads to obesity and the metabolic syndrome. Ann N Y Acad Sci 1148:232-237.

Ripke S, Wray NR, Lewis CM, Hamilton SP, Weissman MM, Breen G, et al., Major Depressive Disorder Working Group of the Psychiatric GWAS Consortium (2013). A mega-analysis of genome-wide association studies for major depressive disorder. Mol Psychiatry 18:497-511.

Maniam J, Morris MJ (2012). The link between stress and feeding behaviour. Neuropharmacology 63:97-110.

Nierenberg AA, Alpert JE, Pava J, Rosenbaum JF, Fava M (1998). Course and treatment of atypical depression. J Clin Psychiatry 59 (Suppl 18):5-9.

Paykel ES (2008). Basic concepts of depression. Dialogues Clin Neurosci 10:279-289.

Petrakis L, Gonzalez G, Rosenheck R, Krystal J (2002). Comorbidity of alcoholism and psychiatric disorders: an overview. Alcohol Res Health 26:81-89.

Petry NM, Barry D, Pietrzak RH, Wagner JA (2008). Overweight and obesity are associated with psychiatric disorders: results from the National Epidemiologic Survey on Alcohol and Related Conditions. Psychosom Med 70:288-297.

Purcell S, Cherny SS, Sham PC (2003). Genetic Power Calculator: design of linkage and association genetic mapping studies of complex traits. Bioinformatics 19:149-150.

Rietschel M, Mattheisen M, Frank J, Treutlein J, Degenhardt F, Breuer R, et al. (2010). Genome-wide association-, replication-, and neuroimaging study implicates HOMER1 in the etiology of major depression. Biol Psychiatry 68:578-585

Sah R, Geracioti TD (2013). Neuropeptide $Y$ and posttraumatic stress disorder. Mol Psychiatry 18:646-655.

Siddiq A, Gueorguiev M, Samson C, Hercberg S, Heude B, Levy-Marchal C, et al. (2007). Single nucleotide polymorphisms in the neuropeptide $Y 2$ receptor (NPY2R) gene and association with severe obesity in French White subjects. Diabetologia 50:574-584.

Singh T, Williams K (2006). Atypical depression. Psychiatry (Edgmont) 3:33-39.

Sinha R, Jastreboff AM (2013). Stress as a common risk factor for obesity and addiction. Biol Psychiatry 73:827-835.

Sullivan PF, Kessler RC, Kendler KS (1998). Latent class analysis of lifetime depressive symptoms in the national comorbidity survey. Am J Psychiatry 155:1398-1406.

Thase ME (2007). Recognition and diagnosis of atypical depression. J Clin Psychiatry 68 (Suppl 8):11-16.

Wei Z, Zhang K, Wen G, Balasubramanian K, Shih PA, Rao F, et al. (2013). Heredity and cardiometabolic risk: naturally occurring polymorphisms in the human neuropeptide $\mathrm{Y}(2)$ receptor promoter disrupt multiple transcriptional response motifs. J Hypertens 31:123-133.

Wetherill L, Schuckit MA, Hesselbrock V, Xuei X, Liang T, Dick DM, et al. (2008). Neuropeptide $Y$ receptor genes are associated with alcohol dependence, alcohol withdrawal phenotypes, and cocaine dependence. Alcohol Clin Exp Res 32:2031-2040. 